\title{
Lapurdum
}

Euskal ikerketen aldizkaria | Revue d'études basques |

Revista de estudios vascos | Basque studies review

$5 \mid 2000$

Numéro V

\section{Critique raisonnée d'un compte rendu déraisonnable (bis)}

Jean-Baptiste Orpustan

\section{OpenEdition}

\section{Journals}

Édition électronique

URL : http://journals.openedition.org/lapurdum/1362

DOI : 10.4000/lapurdum.1362

ISSN : 1965-0655

\section{Éditeur}

IKER

Édition imprimée

Date de publication : 1 octobre 2000

Pagination : 393-404

ISBN : 2-84127-161-7

ISSN : 1273-3830

Référence électronique

Jean-Baptiste Orpustan, « Critique raisonnée d'un compte rendu déraisonnable (bis) », Lapurdum [En ligne], 5 | 2000, mis en ligne le 01 juin 2009, consulté le 21 septembre 2020. URL : http:// journals.openedition.org/lapurdum/1362 ; DOI : https://doi.org/10.4000/lapurdum.1362 


\title{
Critique raisonnée d'un compte rendu déraisonnable (bis)
}

\author{
Jean-Baptiste Orpustan \\ Professeur de langue et littérature basques à l'Université de Bordeaux III \\ Directeur de la revue Lapurdum
}

UMR 5478

\section{Préalables}

Ce titre demande deux explications préalables :

1. Le compte rendu « déraisonnable» dont le présent article fait la critique « raisonnée "), c'est-à-dire sans excès, au-delà de la justesse, ni danı les termes ni dans les appréciations, est celui de La langue basque au Moyen Âge (IX'-XV' siècles) (Izpegi 1999) que vient de publier, sans doute après une lecture trop rapide et peu réfléchie car le sujet du livre, à savoir la description globale des faits de langue, en phonétique, grammaire et lexique, y est à peu près totalement évacué après le rappel initial des chapitres, Monsieur P. Salaberri Zaratiegi dans la Revista Internacional de Estudios Vascos, 44, 1, 1999, XXX-XXX p. 20-24.

2. Le « bis » du titre indique que la présente critique reprend ou continue, sans répéter le détail de l'argumentation, ce qui fut déjà exposé dans un article paru sous le titre « Remarques sur les Observaciones en torno a la obra Toponymie basque de J.-B. Orpustan, intitulé nouveau: Sobre toponimia del País Vasco Norpirenaico d'Alfonso Irigoyen (Bilbao septembre 1990) » dans le Bulletin du Musée Basque, $n^{\circ} 131,1^{\text {" }}$ semestre 1991, p. 11-30. M. P. Salaberri a dû oublier ce texte, ou bien il l'a ignoré comme il l'ignorait lors de sa thèse de 1992, ce qui disqualifie d'emblée une bonne part sinon la totalité, au moins au plan des principes généraux qui s'imposent à tout chercheur tant soit peu consciencieux, de sa très sommaire critique. Mais il n'ignore pas du tout les travaux de toponymie basque d'A. Irigoyen, y compris celui qui vient d'être cité ; et il ne ménage pas son admiration pour l'auteur, sûrement avec raison du reste, car il faut apprécier un ouvrage particulier à l'aune de l'œuvre entière, et celle de l'auteur en question a incontestablement marqué le champ des études basques dans la seconde moitié du $\mathrm{XX}^{e}$ siècle. Il peut ainsi se référer à lui lorsqu'il écrit, réduisant le projet de l'ouvrage critiqué à la seule question de l'étymologie des toponymes médiévaux (traduit de l'espagnol comme l'ensemble des citations) : «L'étymologie n'est pas l'un des points forts du chercheur 
bas-navarrais, comme il l'a déjà démontré dans son ouvrage Toponymie Basque de 1990 (Presses Universitaires de Bordeaux) qui fut critiqué avec âcreté (sic : "acritud") par Alfonso Irigoyen dans Sobre toponimia ... etc. » (p. 21). Il faut pourtant rappeler que lia nette tendance d'A. Irigoyen à verser dans l'anthropocentrisme quasi systématique pour expliquer les toponymes basques - car tout le monde sait qu'il y a, à côté de noms de lieu d'étymologie encore très incertaine sinon inconnue, des toponymes à base anthroponymique clairement démontrable dans les territoires basques, quoique beaucoup moins nombreux que dans les zones extérieures - reçut en son temps de sérieuses critiques de la part de personnalités particulièrement compétentes en la matière.

Auparavant M. Salaberri avait exprimé son étonnement de ne pas voir cités dans l'ouvrage " une série d'articles et livres qui sont fondamentaux dans les études de toponomastique basque comme par exemple la plus grande part de l'œuvre d'Alfonso Irigoyen - il mentionne seulement un article de celui qui fut académicien biscaïen - et il ne cite pas d'autres travaux que cependant il a utilisés » (p. 20). Mais c'est effectivement là le parti-pris énoncé dès le départ pour un ouvrage de synthèse-mais M. P. Salaberri a-t-il appris, depuis 1992, ce qu'est et ce que doit être la synthèse ? -, qui s'appuie sur les textes fondamentaux, non particulièrement et seulement de la toponomastique, mais touchant à la linguistique historique basque générale d'une part (d'A. Luchaire à l'œuvre en tous points essentielle de L. Michelena), et d'autre part et avant tout sur les recueils de textes et citations, et non sur leurs commentaires particuliers : contrairement à ce qu'écrit trop vite M. Salaberri, ce sont seulement ces ouvrages-là, avec de plus les travaux cités en notes, qui « ont été utilisés ». Ces recueils, qui contiennent sinon tous, du moins la plupart des références, dates et éventuellement commentaires et traductions (ou comme le dit l'auteur « étymologies ») connus à ce jour sur le basque médiéval, constituent, avec les ouvrages précédents, la rubrique «Eléments de bibliographie critique » en tête de La langue basque médiévale (p. 12-15) après l'introduction : cette rubrique d'ouvrages tous publiés ou accessibles a été mise là pour n'avoir pas à alourdir le texte déjà compact par des milliers de notes répétitives. Et si l'on n'y trouve pas le nom d'A. Irigoyen, c'est que, à côté de bien des analyses de détail pertinentes, et quelques points nécessairement discutables (ou même objectivement inexacts, comme souvent en toponymie, basque ou autre, y compris dans mes propres travaux bien sûr et ceux de M. Salaberri), ce n'est pas dans ses recueils d'articles (qui forment la série des De re philologica linguce vasconicae etc.) que sont réunies et ont été prises les citations médiévales importantes pour l'histoire de la langue basque. Mais on le trouvera d'abondance, pour tel ou tel point particulier, comme celui des autres commentateurs, dans les ouvrages cités : par exemple, si le nom d'A. Irigoyen n'était cité qu'une fois (p. 40) parmi 147 titres dans le très vaste (pour le territoire ibérique) et précieux recueil des Terminos vascos en documentos medievales de los SS. XI-XVI de J. Arzamendi (Bilbao 1985) qui a été la principale source des citations médiévales en territoire ibérique pour La langue basque au Moyen Âge... (p. 12), dans un autre travail également cité sous la même rubrique (p. 15), à savoir la thèse du propre $P$. Salaberri dans la version première dont je dispose (p. 1887-1888), où il apparaît 13 fois (ce qui est moins 
que les 25 titres de J.-M. Jimeno Jurio, il est vrai onomasticien navarrais touchant de plus près au sujet de la thèse, ibid. p. 1889-1890). Car, je dois le rappeler en passant, en tant que spécialiste de l'onomastique basque ancienne très antérieurement à M. Salaberri comme pour la pratique et la connaissance du basque, j'étais membre de son jury de thèse à côté précisément d'A. Irigoyen et d'autres collègues d'Espagne et de France le 13 novembre 1992 à l'Université de Vitoria. J'avais fait remarquer alors que $\mathrm{M}$. Salaberri qui citait 4 de mes titres parus à ce moment-là (p. 1894-1895), aurait pu en citer 7 autres, il est vrai publiés au Bulletin du Musée basque, que visiblement il ignorait alors comme aujourd'hui, sans pour autant lui en tenir, ni pour d'autres insuffisances inévitables dans une thèse, la moindre rigueur. Il est tout de même bon de rappeler encore que ce Bulletin sous la direction de J. Haritschelhar, fut pendant de longues années la seule revue publiant régulièrement en France des travaux sur le domaine basque, linguistiques et autres, à caractère scientifique.

\section{2. "A prioris ", phobies et " phylies" (traduction de la formule " fobias y filias ») en phonétique}

Allant bien au-delà des formules que son maître en toponymie A. Irigoyen utilisait dans sa très personnelle critique de la Toponymie basque, M. P. Salaberri a résumé dans ces trois termes les « défauts capitaux 》, "spécialement dans le champ de l'étymologie » (p. 20) qu'il a cru trouver dans la Langue basque au Moyen Age. Certes chacun de nous porte en lui ses origines culturelles, y compris linguistiques, comme ses choix intellectuels et esthétiques : et rien ne sert de cacher que le BasNavarrais que je suis, né dans un milieu encore à peu près exclusivement bascophone " depuis toujours » et « vieux Basque » donc, qui apprit, comme la plupart en ce temps-là, sans télévision ni guère de radio au fond des campagnes, à parler français à l'école à partir de l'âge de 4 ou 5 ans, et dont les références en langue basque sont depuis toujours celles du «navarro-iabourdin » classique, depuis nos premiers écrivains classiques des XVI-XVII siècles, jusqu'aux Etchepare, Barbier ou Lafitte du $X X^{e}$ (un état de la langue basque «élaborée et littéraire», pour le dire en formule simple, que je ne vois nullement et nulle part surpassé au tournant du XXI siècle, ni même, le plus souvent, atteint), a ses préférences et a fait depuis longtemps ses choix.

Mais dans la mesure du possible, c'est-à-dire du conscient, ils n'ont pas part à la pure description raisonnée d'un état, ou plus exactement des éléments d'un état de langue : en l'occurrence un basque " médiéval » ou un état médiéval de la langue basque aux contours géographiques et historiques tout compte fait bien lâches et même assez largement approximatifs, en tout cas très incomplet par rapport à ce que fut la réalité. Il serait ridicule de prétendre penser autrement, ce pourquoi un ouvrage de synthèse comme La langue basque... ne peut avoir pour objet de dresser une carte des dialectismes médiévaux, qui serait du reste nécessairement très lacunaire et même irréalisable, même s'il en signale, en cours de route, quelquesuns. Mais pour autant, ce n'est pas dans La langue basque médiévale que M. Salaberri, qui regrette que cet ouvrage soit rédigé avec une « suprenante ("asom- 
brosa") allégresse et légèreté »-je le trouve pour ma part fort aride ! - et non sans doute à coups de discussions pesantes, accumulatoires et répétitives - sait-il qu'on n'a jamais fait mieux qu'au XVIII siècle pour écrire le savoir en bon français, puisque français il y a ? -, doit aller chercher mes préférences linguistiques, ou mes « phobies » ou allergies, non " a prioristiques » pourtant, car même l'esthétique peut se fonder en raison et doit même chercher à le faire. Je lui en indique la voie, quitte à le mettre encore plus mal à l'aise, peut-être, quant à ses préférences à lui. En plus des études et commentaires ponctuels axés sur quelques traits de langue aujourd'hui ou naguère à la mode mais à l'évidence déviants par rapport à l'authenticité linguistique traditionnelle que je vois (certes à côté de bien des insuffisances, comme partout) dans mon domaine dialectal exposés dans quelques conférences et articles, il les trouvera principalement dans les deux ouvrages que j'y ai consacrés : au plan de la simple pratique langagière fondée sur l'analyse grammaticale, et centrée sur l'écrit élaboré et de caractère littéraire mais sans rompre pour autant avec le parlé vécu que j'ai entendu et appris autour de moi, dans Basque et Français, méthode abrégée de traduction (Izpegi 1997), et pour une pratique systématique des formes et formules d'une langue poétique, dans les poèmes strophiques de Jean de Sponde traduits dans Ezponda (1557-1595) euskal neurthitzetan (Izpegi 1995). Et pour une appréciation stylistique, donc délibérément esthétique cette fois de l'écrit basque, il verra où vont mes choix - avec peu de vraies «phobies»! - dans le Précis d'histoire littéraire... (Izpegi 1998).

Est-ce que les traits de la langue médiévale correspondent ici ou là à ceux de ce basque littéraire élaboré de notre tradition, selon la formule habituelle, navarrolabourdine, ou s'en éloignent? Peu importe à vrai dire, et les correspondances ou les différences, si elles existent, ne tirent pas à conséquence, d'autant moins que les fragments épars, en eux-mêmes inégalement nombreux (d'où la différence en extension des divers chapitres), de phonétique, syntaxe, morpho-syntaxe et lexique rapportés dans l'ouvrage, comme l'indique M. P. Salaberri en tête de son compte rendu, mais sans s'intéresser pratiquement à rien d'autre que quatre ou cinq points mineurs de phonétique et quelques exemples de ce qu'il nomme très approximativement « étymologie », sont fort loin de donner une idée de la langue basque littéraire médiévale, si toutefois elle exista hors des chants de circonstance et autres dont il ne reste presque rien, et pratiquement rien qui reproduise vraiment et avec précision, sans altérations postérieures inévitables, les textes primitifs. Raison déterminante pour laquelle ont été écartés tous les fragments d'origine médiévale collectés à la fin du XVI ${ }^{e}$ siècle, à plus forte raison les textes postérieurement écrits. Mais si, chose bien connue par ailleurs, je trouve que l'aspiration était générale encore $\mathrm{au} \mathrm{XI}$ siècle et à plus forte raison avant (quoique l'ouvrage ne prétende à aucune reconstruction et se limite à analyser les citations), à peu près comme elle l'est restée dans mon... bas-navarrais méridional natal, si la séquence consonantique apicale-sifflante donne presque dans tous les cas des fricatives infiniment plus douces à l'oreille et à la prononciation (Larramendi trouvait tant de douceur encore dans son guipuscoan du début du XVIII siècle !) que les affriquées que M. P. Salaberri et d'autres, très "philo-affriquées "-trop à mon goût, mais là n'est pas la question -, voudraient imposer partout, comme s'il fallait refaire une langue 
déjà faite depuis des millénaires, mais certes améliorable quoique peu changeante de nature depuis longtemps, et d'autres faits semblables, tant mieux ou tant pis : c'est un fait. Il faut se rendre, là comme ailleurs, à la raison, qui n'est pas forcément ou seulement le « sens commun » que dit M. Salaberri (p. 20 : s'il ne tenait qu'au « sens commun » la terre tournerait toujours autour d'un minuscule soleil), c'est-à-dire au fait, archidémontré et indiscutable malgré toutes les incertitudes dans la réalisation graphique médiévale des sifflantes basques : la langue moderne, par rapport à l'ancienne, tend au développement des sifflantes affriquées au détriment des fricatives comme des apicales au détriment des dorsales. Et il est vrai, par ailleurs, que je n'y vois pas une amélioration dans l'équilibre sonore, donc esthétique, général de la langue, bien au contraire.

Le scepticisme de M. Salaberri, très légitime dans tout ce qui touche à l'interprétation des graphies médiévales, tourne au préjugé irrationnel, quand il n'accepte pas que I'on puisse, à partir de 19 citations de toutes dates et toutes époques alignées à la page 117 (et le point de suspension indique qu'on aurait pu en ajouter beaucoup d'autres, infiniment plus que d'affriquées dans la même position), conclure «qu'au Moyen Âge c'est normalement la fricative qui suit les apicales » et que « (l') affriquement ou du moins son caractère automatique est un trait récent dans l'histoire de la langue $\gg:$ s'il y a un a priori à ce sujet, c'est dans l'esprit de M. Salaberri.

Un autre point qui chiffonne beaucoup M. P. Salaberri c'est la description de la palatalisation «hypocoristique ». Mais, comme déjà A. Irigoyen tenait à prendre des hypothèses que j'affirmais avec insistance comme telles pour des certitudes et des affirmations (voirl'article du Bulletin de 1990 déjà cité où la question est exposée en détail), il ne sait pas lire la nuance là où elle est, et même dans sess propres citations : « hypocoristisation à outrance, signe peut-être d'une certaine dévalorisation de la langue populaire face au castillan officiel» (p. 21). Cette « hypothèse prudente » que le développement des formes hypocoristiques, que je trouve, après d'autres, concentrées dans la documentation alavaise de la fin du XV siècle davantage qu'ailleurs dans la documentation connue et publiée de même époque, et bien qu'il y ait aussi partout et depuis les toutes premières citations nombre d'hypocorostiques bien signalés dans le livre, "peut » indiquer un état de langue peu ou prou en cours de « dévalorisation » face au castillan officiel déjà très prédominant, dans une zone où l'usage du parler basque sera bientôt abandonné, et sans « peutêtre » (mais pourquoi pas en partie ?) que ceci soit la conséquence directe de cela, ne me paraît pas absurde du tout. La réduction ou le maintien, ce qui revient au même lorsque la langue officielle progresse, du langage régional aux modes de parler du cercle familial et même enfantin, qui se poursuit avec la prolifération aujourd'hui des hypocoristiques dans toute l'anthroponomastique basque, comme la fréquence des onomatopées au détriment des lexèmes «étymologiques 》, comme l'intrusion des romanismes qui finissent par faire oublier les mots basques (le cas de borta pour athe " porte ", que j'ai vu moi-même s'imposer progressivement dans l'usage, est relevé par M. Salaberri p. 22, mais pourn'y voir, lui, aucun inconvénient) ou même, ce qui est pis aujourd'hui, les structures syntaxiques, est objectivement l'un des signes visibles de l'état de dévaluation de la langue locale 
face à la primauté de la langue officielle. Trouver de plus en plus de palatalisations hypocoristiques « à mesure qu'on recule dans le temps dans les zones où le basque a été la langue majoritaire etc. » (p. 21) mais... où elle s'est ensuite perdue, contredit-il en quoi que ce soit l'appréciation qui précède ?

Le fait que la langue officielle fut le castillan en zone « ibérique » ou « hispanique » (Iberia, Hispania sont des mots qui nomment tout le territoire au sud des Pyrénées depuis bien plus de 2000 ans... et je les trouve de très loin les plus aptes à dire les espaces bascophones anciens et modernes, car en termes objectifs la Biscaïe n'est pas « au sud », par exemple, ni les pays de Cize et Baïgorry " au nord »!) quatre ou cinq siècles avant le français en zone aquitaine, qui n'y est vraiment en force qu'à partir du XVII et surtout du XVIII siècle, est irréfutable. Je ne vois donc rien d'étonnant, c'est le contraire qui le serait, dans l'influence castillane sur l'onomastique basque ibérique dont je donne maint exemple mais que M. Salaberri semble récuser (p. 21), se référant à la page 132 où il est dit, à propos des affriquées initiales, que la sifflante palatale initiale de xipi en graphie médiévale partout constante chipi, est « affriquée dans le domaine hispanique conformément à la phonétique castillane, fricative en domaine aquitain ». Si l'extension de l'affriquement palatal en sifflante initiale n'était pas l'une des nombreuses marques de cette influence, pourquoi le basque $n$ 'a-t-il nulle part d'affriquée non palatale en position initiale, et à quoi attribuer cette exception remarquable? On ne peut donc prétendre, au simple vu des textes, que la phonétique castillane - ou « navarro-castillane » ou "navarro-aragonaise » si l'on veut pour le « roman navarrais » qui n'en est qu'une variante pour l'essentiel assez peu différenciée - ne transparaît pas dans l'écriture des noms basques et n'a pas eu d'influence sur la phonétique basque ibérique, et depuis des siècles (dès le $\mathrm{XVII}^{\circ}$ siècle où elle naît en espagnol la jota passe aux dialectes hispaniques navarrais et guipuscoan sauf au biscaïen), tout comme le français en a eu, mais beaucoup plus tard (la vibrante « uvulaire » entrée en français à partir de la fin du XVIrr ${ }^{e}$ siècle, ne commence à passer en basque qu'au cours du XX ${ }^{e}$ siècle), sur lies dialectes aquitains. Cette influence apparaît encore mieux dans les transcriptions des noms de maisons des provinces aquitaines au XIV siècle (Les noms des maisons médiévales en Labourd, Basse-Navarre et Soule, Izpegi 2000).

S'agissant de phonétique encore, M. P. Salaberri relève dans son étrange " compte rendu ", outre une citation (qui ne peut être que " de langue basque ") arronkari pour «Roncal » d'où il prétend étrangement et abusivement déduire sans doute que j'ignore le nom usuel moderne et sans doute déjà médiéval de cette province Erronkari (p. 21), des faits comme ceux-ci :

pour 1108 larrahona donné comme un des exemples du mot on/hon «bon" (p. 79) et interprété ainsi comme il le rappelle par L. Michelena (p. 22), il prétend récuser cette analyse au prétexte que ce lieu du val d'Amescoa « se disait Larragoa » ("se decía ») : mais quand donc ? et a-t-il quelque attestation médiévale de cette forme? si oui il fallait la donner; car ou bien il y a eu double tradition et double étymologie, non attestée pourtant au Moyen Âge, ou le -goa final est issu de l'ancien -hona, ce qui n'est pas phonétiquement tout à fait indémontrable à partir des éléments connus de la phonétique basque médiévale ; il est vrai 
que " on » tant qu'il est compris comme « bon » ne perd pas sa nasale, mais le propre de la toponymie c'est justement de devenir vite sans signifié dans l'usage et la conscience populaire, fait archi-connu; et alors tout segment identique suit les mêmes changements phonétiques ;

pour le mot lur(r) " terre " (je mets à dessein le signe de vibrante forte entre parenthèses), au vu de ses formes médiévales, mais aussi de la série incontestablement proche qu'il fait en basque avec ur « eau » et zur «bois (matériau) », arguments que M. Salaberri se garde bien de citer, le texte dit ceci : « il est permis de penser que ce mot a fait partie [...] d'une série à vibrante finale faible » (p. 132), permission prudente sur une question difficile d'étymologie vraie et de variation très certaine dans les vibrantes finales (affaiblissement ou renforcement ? l'étonnant serait ici aussi que cette opposition finale, qui n'est pertinente linguistiquement que pour le couple ur/urr alors qu'elle l'est partout sans exception, comme attendu, en position intervocalique, fût restée immuable en tous lieux au cours des siècles : en Soule on dit bien $(h) o(r) a$ « le chien » avec élimination actuelle de la vibrante " faible ", mais en Basse-Navarre ihiztorra " le chien de chasse " composé sur le même !), mais que M. Salaberri ne m'accorde pas, disant pourtant plus prudemment cette fois que ce n'est pas « l'explication la plus idoine» (p. 23) ! En quoi, si l'on ne donne pas l'explication « idoine », cette remarque est-elle utile ?

Pour les occlusives en " position combinatoire», sourdes et au besoin assourdies après sifflantes (et sonores ou sonorisées après nasales et latérales, du moins dans les emprunts anciens et dans les zones les moins en contact avec la prononciation romane), trait constant de phonétique basque parfaitement observable du plus haut Moyen Âge à l'époque contemporaine, M. Salaberri met en doute que la prononciation correspondant à la fameuse citation de San Millán ezdugu ait été réellement eztugu (p. 23) et du même coup que les citations médiévales comme 1025 arrozuide etc. avec sonores, ultra-minoritaires dans l'ensemble des citations médiévales, procèdent, comme il apparaît pourtant très incontestablement, d'une " décomposition analytique des mots véritablement composés chez certains scribes " (p. 91) : de même que toute suite de nasale-occlusive bilabiale ne peut phonétiquement se réaliser, en basque comme ailleurs, en nasalisant la voyelle précédente que par une bilabialisation de la nasale - $m b / m p$ - quels que soient l'arbitraire et même l'absurdité de la pratique écrite du basque moderne en la matière, de même la sifflante (sourde en basque) impose l'assourdissement " automatique " ; à moins de détachement et d'arrêt de l'émission de voix, ce qui ne se produit ni en « composition " s'il y a effectivement composition, ni en préfixation dans le cas du verbe négatif. Mais M. Salaberri s'intéresse moins en apparence à la logique interne de la langue, médiévale ou autre, qu'à essayer, sans (se ?) le dire évidemment, de justifier une écriture actuelle du basque, bien illogique sur ce point comme il a été souvent souligné, née à l'époque post-classique sur le modèle latinoroman où la négation reste un morphème indépendant, et étendue tardivement à l'usage écrit, diversement suivi du reste et avec raison par les poètes et prosateurs. Essayer de contredire ces réalités c'est amener de mauvaises raisons à l'appui d'une mauvaise cause. 
Dans le cas du nom navarrais ancien de Aoiz, en basque actuel Agoitz, mais écrit dans l'une des plus anciennes citations du XI ${ }^{e}$ siècle 1079 ahoiz, en zone où pourtant l'aspiration se perd très tôt, puis plus irrégulièrement 1257 haoyç indiquant " peut-être » comme très souvent que l'aspiration primitive n'est pas entièrement perdue mais qu'on ne sait plus la représenter correctement, tout en n'excluant pas que l'aspiration, comme souvent en zone hispanique, ait pu être remplacée par l'occlusive (p. 84) (« es posible que así sea », p. 22), M. Salaberri choisit l'étymologie d'A. Irigoyen. Celui-ci proposait d'y voir le résidu d'un hypothétique Aionis et le rapprochait de Ayesa écrit Ageza, alors qu'il n'y a, me semble$\mathrm{t}$-il, aucun témoignage de " *Aioiz »; et comme le passage direct de $-i$ - à $-g$ - serait des plus surprenants en phonétique basque, il imaginait l'apparition d'une consonne " antihiatique » après perte du yod intervocalique ; mais comme l'analyse est, de l'aveu même de l'auteur, hypothétique ( « sería » p. 22), et sans entrer dans l'étymologie du nom, sur laquelle je n'ai guère d'opinion et celle d'Irigoyen me semblant très hasardeuse, voilà encore une remarque vaine et, pour le moins, peu utile à la compréhension du texte commenté.

\section{Analyses et " étymologies"}

Tant mieux si M.P. Salaberri a fait, lui, « son point fort » de l'étymologie basque et toponymique en particulier ; mais à vrai dire je n'en crois rien, même s'il a sûrement fait des progrès depuis le temps de sa thèse, parce que, jusqu'à présent et quoique bien des sottises soient progressivement rectifiées et des ombres éclairées par les travaux de chercheurs à la fois audacieux et rigoureux, il n'y a personne pour y être parvenu vraiment. Et la chose est encore plus aléatoire pour certains toponymes anciens. Les citations d'onomastique constituant comme l'on sait non seulement la majorité, mais la presque totalité (plus de $80 \%$ bien que le calcul exact ne soit pas établi) du corpus des citations médiévales basques prises dans leur ensemble, l'incertitude est le signe premier, et parfois constant, de nombre d'analyses. Mais il y a de quoi s'étonner quand M. Salaberri commence par noter que la description de La langue basque au Moyen Âge... « se fonde presque exclusivement sur des faits d'onomastique médiévale [...] quoique sporadiquement (il soit) tenu compte aussi d'autres apports » (p. 20) : connaît-il donc d'autres textes basques médiévaux datés que ceux qui sont utilisés dans l'ouvrage? S'il en connaît, ce qui est plus que douteux, il devait les signaler.

Une fois le corpus constitué, tel qu'il est et à peu près exhaustif pour les citations connues jusqu'en ces dernières années, avec peu de chances d'en trouver de nouvelles de quelque importance, il faut l'analyser. Ce n'est pas là essentiellement une question d'étymologie, bien qu'elle y ait sa part aussi, heureusement faisable pour pour la grande majorité des composés (sinon des composants !) cités et reconnaissables comme appartenant, à un titre ou à un autre, à la langue basque. Que le nom d'Armendaritz en Basse-Navarre soit anthroponymique ou toponymique, peu importe pour le projet du livre; et la question a été déjà suffisamment débattue en 1990. Mais M. Salaberri l'ignore ou n'en veut rien savoir, et il se croit autorisé néanmoins à condamner l'explication traditionnelle bien connue de ce nom-expo- 
sée dans divers ouvrages très antérieurement à ma Topoymie de 1990 ! - et à la présenter comme une maligne entreprise de tromperie dirigée par moi contre lui et ses amis (" (il) tenta de nous faire croire que... » sic et resic !). Et pourtant, l'étymologie sur « base anthroponymique " de ce nom bas-navarrais, qui en fait un doublet bien étrange du très nordique Armentières, reste toujours aussi loin d'être «claire »qu'il l'assure imprudemment (p. 22), même si, un siècle avant A. Irigoyen, A. Luchaire l'avait déjà exposée en... 1881 ! Et sa forme depuis longtemps attestée (toujours sans le - $e$ final postiche d'aujourd'hui) suffit pour en tirer des informations utiles à la connaissance de la linguistique basque médiévale. Et il en va à peu près ainsi pour le reste.

Les autres critiques que M. Salaberri développe au sujet de ces «étymologies » ou plutôt de ces analyses sont-elles mieux fondées, et remettent-elles sérieusement en cause le projet de l'ouvrage ? On en jugera par quelques exemples, sans s'attarder sur les noms comme Beraskoitz, Berastegi etc. (p. 22) déjà traités en 1990 et sans qu'il y ait rien de nouveau à ajouter depuis lors quant aux meilleures hypothèses d'analyse, ni sur l'origine du nom de la Navarre, l'ancien nabarra dont dérive par latinisation nabarrus «navarrais » et de celui-ci par romanisation le médiéval castillan navarro d'où sort évidemment le nafarro moderne : sachant que la consonne labio-dentale ne fait pas partie du consonantisme basque primitif et rarissime encore au Moyen Âge, absent jusqu'à l'époque moderne en biscaïen, M. Salaberri voudrait-il en faire l'étymon premier ? Il est vrai que cette forme hispanique est déjà chez des écrivains labourdins ou souletins classiques, mais non chez tous (Tartas par exemple etc.) ni dans l'usage populaire local (correspondance labourdine du XVII siècle), sinon pour désigner le "vin de Navarre »: son « siempre » est un " présupposé » et une erreur, tout simplement ;

a) à propos des composants du toponyme zilegieta (p. 21) il donne un résumé tendancieux du texte en affirmant que ce nom cité depuis le IX $\mathrm{X}^{\mathrm{e}}$ siècle est considéré dans l'ouvrage " comme dérivé de zilo [...] et non comme dérivé de zilegi "monts communaux » selon Azkue » etc., alors que le texte (p. 61) donne cette explication comme préférable (" plutôt que ") après réflexion et en tenant compte de toutes les formes dialectales, sur celle, traditionnelle, à partir de zilegi « permis », la référence au dictionnaire d'Azkue qui collige ses mots à la fin du XIX' siècle étant de toute façon peu utile pour le... IX !

b) urru composant des noms bas-navarrais médiévaux Aldacurru, Mendicurru et présent dans bien d'autres n'a probablement rien à voir avec le mot salazarais (avec attestation médiévale ou non ?) urru au sens de «sommet rond» (p.22), qui se dit dans cette zone par le paronyme murru dans le lexique médiéval et la toponymie quand ce n'est pas par le latinisme kurku;

c) il suffit d'un peu de logique et de connaissance du lexique botanique pour penser que la base goro- de la toponymie a été à peu près sûrement « houx » et s'est allongée ensuite par suffixes successifs pour faire dès le Moyen Âge gorosti qui devait être donc " houssaie » en effet comme indiqué dans le glossaire : voilà qui n'a rien de spécialement comique ( «lo mejor es que ... »p. 23) à condition de savoir comment s'organise le lexique botanique en toponymie ou ailleurs (iratze à l'ori- 
gine « fougeraie » qui a fini par remplacer dans nos dialectes la base médiévale ira " fougère " au même sens par exemple ; et peut-être les modernes mahasti « vigne» et mahats " raisin » issus de l'emprunt latin mina ou miña " vigne " seul en usage au Moyen Âge à côté de ardan et ardantze). Si du reste, comme semble le vouloir M. Sallaberri, il fallait y voir goroa " couronne » on ne voit pas pourquoi, à part le goroabe de Pampelune (1408 goroave), le - $a$ disparaîtrait d'emblée pour faire goroeta et non par exemple un "goroaeta évidemment non attesté, puisque ce suffixe n'élimine pas les voyelles finales organiques; mais ce goro-là pas plus que tous les autres termes cités « dans le reste de l'œuvre» (ibidem) n'a, en toute rigueur et tradition linguistiques, aucun besoin d'astérisque ; ce serait même une faute ridicule, puisque, loin d'être une reconstruction, c'est un élément bien réel et bien attesté du lexique médiéval. Ce qui est incertain dans une certaine mesure, c'est seulement le sens qu'on peut lui prêter ; mais le mot gorost $i$ avec ses trois syllabes, si ce n'est pas un emprunt, ce qui n'a pas encore été démontré, ne peut qu'être, lui, un dérivé suffixé ; et il s'agit évidemment d'un fait de morphologie lexicale ;

d) la voyelle finale $-a$ de nombre de toponymes, et hors noms de maisons où la détermination est de règle, a été probablement un déterminant tant que les noms de lieux descriptifs ont été des noms « communs », et M. Salaberri reconnaît avoir " (lui-même) défendu cette possibilité » (p. 23), en quoi il a eu sans doute raison, même s'il estime seulement que ce n'est pas « prouvé » : mais quel doute par exemple pour Bizkaia? Il est vrai que dans la liste des toponymes déterminés dans l'usage à la page 162 le nom Donostia apparaît, bien que ces noms ne soient pas donnés comme citations médiévales : le fait que la forme originale citée par L. Michelena Donasabastiai implique qu'il a pu avoir à l'origine un - $a$ organique n'empêche pas que ce nom est très souvent écrit aujourd'hui (comme autrefois : Donostian Donosti... dit le proverbe !) au nominatif Donosti parce que cet $-a$ est pris pour déterminant, tout comme les dérivés en -eria sont souvent écrits et dits -eri, bilzar au lieu de bilzarre, etc., avec autant de voyelles organiques qui tombent ; si l'on ne cite pas non plus Iruñea (p. 20) aujourd'hui officialisé avec son déterminant, c'est d'une part que cette liste n'est pas exhaustive et qu'il y a aussi depuis longtemps des lieux où l'on continue à dire aussi bien Iruñe qu'Iruña et jamais Iruñea ;

e) la suffixation nominative en - $r a$ de quelques noms à $-a$ final organique, depuis longtemps relevée par un René Lafon dans la littérature des XVI-XVII siècles, est parfaitement « archaïque » déjà au Moyen Âge et j'avoue ne pas l'avoir trouvée ou reconnue dans la documentation médiévale consultée et indiquée en tête du livre, comme dans la littérature postérieure, qu'en "zone aquitaine " (p. 164) ; mais il ne me choque pas du tout de savoir qu'elle a été utilisée en salazarais (p. 23), au contraire, qui aurait eu alors - mais est-ce si étonnant ? - des éléments aujourd'hui " dialectaux » communs avec l'aquitain (Aquitania est comme Hispania, Iberia, un nom territorial vieux de plus de 2000 ans); et si l'on en a aussi des témoignages médiévaux, qui ont pu m'échapper mais que M. Salaberri à le tort de ne pas donner et de ne pas dater, on ne voit pas en quoi cela affaiblirait une description globale du basque médiéval, qui n'est pas et ne peut être, il faut le répéter, un atlas dialectal détaillé ; 
f) le suffixe collectif -doi/-dui et ses variantes s'ajoutent dans la très grande majorité des cas et partout à des noms végétaux nombreux ; "rares » sont en effet (p. 24) les bases non végétales ainsi suffixées, parmi lesquels j'ai cité hardoi et lapiztoi (p. 266) ; mais cette formulation ne convient pas à M. Salaberri, qui y ajoute après avoir cité (deux fois) le premier de ces noms, une série qui désigne de même des noms du même réseau sémantique, sable, pierre, gravier, lauze etc. areadui, arridi, kaskalludi, legardi, lauzadi « par centaines en toponymie », mais sans dire d'abord si c'est en toponymie médiévale et sans les dater, et ensuite sans montrer s'il s'agit bien toujours de - $d i$ issu de -doi et non de quelque autre élément, ce qui rend encore la contradiction (a-t-il compris que « rare » est à prendre au sens relatif à l'ensemble documenté au Moyen Âge ?) a peu près inopérante et pratiquement inutile ;

g) la consonne dite faute de mieux « de liaison » $-l$ - (au lieu des habituelles occlusives dans cette fonction morphologique) est difficilement récusable et M. Salaberri ne le fait pas, tandis qu'il conteste (p. 22) que la nasale - $n$ - beaucoup plus rare ( $\mathrm{R}$. Lafon essaya en vain naguère de comprendre le segment -naga de liginaga) puisse se trouver dans quelques noms, dont le zaldinuaga, et zaldinaga, zaldunaga qui sont de son domaine navarrais historiquement dialectal (p. 39, 46, 345 etc. de sa thèse, où j'avais déjà signalé le rapprochement avec le toponyme souletin) : trois formes qu'il est raisonnable de ramener au même, et sans doute à une base zaldu plutôt que zaldi (que je ne cite pas comme il le croit); encore que, vu justement la "rareté » de ces formes, ce soit une fois de plus un point d'infime détail pour l'ensemble liguistique médiéval.

\section{Des prémisses faibles, discutables ou erronées à une sommaire et inadmissible conclusion}

L'argumentation de M. Salaberri contre La langue basque au Moyen Age se révèle ainsi objectivement faible à qui l'examine d'un peu près, incertaine parce que le plus souvent inexacte et imprécise dans les références au texte et dans sa compréhension, tendancieuse parfois. Elle est par dessus le marché « à côté de la plaque ", puisque, à travers nombre de citations médiévales toujours datées (mais non toutes rapportées !) et prises dans les ouvrages cités en référence, c'est la langue basque médiévale que l'ouvrage prétend, certes très incomplètement mais surr des points souvent essentiels, décrire, et qu'il n'a pas pour objectif, comme le laisseraient penser les thèmes choisis par M. Salaberri, de résoudre toutes les énigmes ou les incertitudes étymologiques, nombreuses, de l'ancienne toponymie des territoires de langue basque. Elles resteront longemps irrésolues, mais sur cette question il faut se rapporter à la Toponymie basque (que ni les observations d'A. Irigoyen ni, à plus forte raison, celles de M. Salaberri ne m'ont conduit à modifier) et aux Noms des maisons médiévales en Labourd, Basse-Navarre et Soule. Au total, M. Salaberri fait un « compte rendu » objectivement très mauvais de l'ouvrage, parce qu'au lieu de mettre le lecteur sur piste comme le devrait tout honnête compte rendu, et hors quelques indications sur l'extension du basque en Béarn médiéval, depuis longtemps connue des spécialistes et très accessoire quant au 
fond, il l'égare en ne citant de la manière qui a été dite que des points de détail tout à fait secondaires dans le projet de l'ouvrage. Le lecteur, s'il n'est très prévenu sur ces questions, sera donc floué.

Et pourtant, après ces remarques sur d'infimes détails du livre, scientifiquement pour le moins douteuses, M. Salaberri a le front d'oser une conclusion inconvenante et grossière qui, s'il y avait une raison valable à cela, ressemblerait fort à un règlement de compte, personnel ou autre (p. 24) : " Enfin pour conclure nous voulons exprimer notre préoccupation parce que des travaux de ce type, dans lesquels les idées préconçues valent plus que les faits et le sens commun (bis), peuvent faire penser au lecteur que dans le champ de l'onomastique et de l'étymologie toute chose est bonne, du moment qu'elle est publiée. Il est clair qu'il n'en est rien ».

Eh bien ! ces propos ne sont pas du tout " de sens commun » : ils font seulement penser que la lecture du livre a ébranlé chez leur auteur quelques « présupposés et certitudes » sur la langue basque ancienne (ou même moderne), à moins de penser qu'ils procèdent d'une tout autre inspiration, qui n'a rien à voir avec les faits de langue et d'histoire linguistique. Ils dépassent d'aussi loin ce que les meilleurs spécialistes de linguistique basque seraient en droit d'écrire sur ces questions, qu'ils restent très en dessous des usages de la simple politesse, fút-elle universitaire. Ils suffisent à disqualifier M. Salaberri et à mettre en cause les institutions (l'auteur est membre de la commission d'onomastique de l'Académie de la Langue Basque) et organes de publication (la nouvelle RIEV est éditée par Eusko-Ikaskuntza) au nom desquelles et dans lesquelles il s'exprime. 